\title{
A normalized area-compliance method for monitoring surface crack development in a cylindrical rod
}

\author{
C.Q. Cai, C.S. Shin* \\ Department of Mechanical Engineering, National Taiwan University, No. 1, Sec. 4, Roosevelt Road, Taipei, 10617, Taiwan, People's Republic of China
}

Received 14 June 2004; received in revised form 8 November 2004; accepted 10 January 2005

\begin{abstract}
A Normalized Area-compliance method has been shown to be able to predict the depth of an elliptical surface crack in a cylindrical rod. The method involves a combination of optical surface crack length measurement and specimen compliance measurement. Finite element analysis was employed to show that the specimen compliance has a one-to-one correspondence with the Normalized Area (NA) or Normalized Harmonic Mean Radius (NHMR) independent of crack depth and crack aspect ratio and with the crack shape and specimen dimension for a range of crack and specimen dimensions. Based on the finite element analysis, master curves of Normalized Compliance versus Normalized Area have been generated for surface-cracked rod under tension with ends free or constrained from rotation. The accuracy of crack depth prediction using this method has been verified by fractography of fatigue cracked rods. Moreover, fatigue crack growth behaviour deduced from this crack depth measurement agrees well with the crack growth data obtained from the standard compact tension specimen.
\end{abstract}

(C) 2005 Elsevier Ltd. All rights reserved.

Keywords: Surface crack measurement; Compliance; Fatigue crack propagation; Surface crack

\section{Introduction}

Cylindrical shaped components such as pins, bolts, reinforcement wires and shafts are commonly used in engineering structures. Under repeated loading, cracks may develop at the surface and grow across the section. Recently, it has been shown that surface cracked rod specimen offered a cost-effective way for evaluating fatigue crack propagation properties [1]. Experimental investigation plays an important role in understanding the crack growth behavior, to validate the stress intensity factor solutions [2] and in structural integrity assessment involving these cracks. Owing to the three dimensional nature of the part-through surface cracks, accurate measurement of the crack front profile is often difficult and indirect. Periodic beach marking had been used to reveal the crack fronts [2-4]. Confocal scanning laser microscopy [5] and electromagnetic method [6] can give accurate crack dimension.

\footnotetext{
* Tel./fax: +86886223622160.

E-mail address: csshin@ntu.edu.tw (C.S. Shin).
}

0142-1123/\$ - see front matter (C) 2005 Elsevier Ltd. All rights reserved. doi:10.1016/j.ijfatigue.2005.01.005
The former was able to measure cracks as small as $10-100 \mu \mathrm{m}$. The latter was shown to be accurate to within $0.03-0.17 \mathrm{~mm}$ of the actual depth of a surface crack. However, these kinds of technique cannot offer in situ real time measurement. Potential Drop (PD) technique using alternating current [7-9], direct [10-13] and reversing direct current [14] had been employed to give real time measurement. Since different crack shape and crack depth combination may produce the same potential drop across a particular region, multiple probe sites are generally needed to define the crack profile [15]. These involved expensive equipment and the set-up were susceptible to electromagnetic and other interferences [16,17]. Typical errors involved in these crack depth measurement are 10-20\%. In this work, a simple method involving conventional low cost compliance monitoring plus optical measurement is proposed and will be shown to give a crack depth resolution of $0.03 \mathrm{~mm}$ and an accuracy of $0.08 \mathrm{~mm}$ in practical cases.

\section{Compliance method for surface crack measurement}

Experimental results suggested that surface cracks in cylindrical rods can be adequately described by 


\begin{tabular}{llll}
\hline \multicolumn{2}{l}{ Nomenclature } & & \\
$a$ & depth of a surface crack in mm & $2 L$ & rod length in mm \\
$b$ & semi-major axis of an elliptical crack in mm & NA & normalized area \\
$C$ & characteristic compliance & NHMR & normalized harmonic mean radius \\
CMOD & crack mouth opening displacement at point $\mathrm{O}$ & $2 S$ & surface arc length of a crack in mm \\
& in mm & $X$ & normalized compliance at point B \\
$D$ & rod diameter in mm & $\varepsilon_{\mathrm{B}}$ & back-face strain at point B \\
$E$ & Young's modulus in GPa & $\theta$ & off-axis angle from the centerline \\
& & & \\
\end{tabular}

a semi-elliptical profile $[2,18,19]$. Fig. 1 shows the schematic of such a crack. To uniquely define the crack front, we need information about the crack shape (such as the aspect ratio $a / b$ ) and a crack dimension (such as surface crack length $2 S$ or crack depth $a$ ). Optical measurement can only give the surface crack length. Compliance is an easily measured quantity and it reflects the size of the crack relative to the net-section area. However, the relative crack size and hence the compliance are again a function of crack shape and crack depth. It has been shown that the PD across a surface crack may be uniquely related to a single parameter called the Normalized Harmonic Mean Radius (NHMR) under different combination of crack aspect ratios and crack depths [20]. The NHMR is defined as:

$\operatorname{NHMR}=\int_{\Gamma} \sqrt{\mathrm{d} x^{2}+\mathrm{d} y^{2}} / D \int_{\Gamma} \frac{\sqrt{\mathrm{d} x^{2}+\mathrm{d} y^{2}}}{\sqrt{x^{2}+y^{2}}}$

where $D, \Gamma, x, y$ are respectively, the rod diameter, the integration path along the crack front and the co-ordinates on the cracked area.

The same NHMR parameter will be used in correlating the compliance. Moreover, a Normalized Area (NA) is also introduced and used in correlating the compliance as well. The Normalized Area is simpler to compute than the NHMR and is defined as:

$\mathrm{NA}=4 A_{\mathrm{c}} / \pi D^{2}$

where $A_{\mathrm{c}}$ is the area of the surface crack.

Finite element method as well as empirical calibration has been used to check whether the compliance has a oneto-one correspondence with the NA or NHMR under different crack depths and aspect ratios. Once this is established, a measurement of the compliance of a surface cracked sample will allow the NA or NHMR to be obtained. Together with the surface crack length data, the shape and size of a surface crack can then be inferred.

\section{Numerical analysis of the compliance}

The relations between compliance and Normalized Area or Normalized Harmonic Mean Radius under different crack aspect ratios, crack depths, specimens Young's modulus and dimensions were analyzed by the finite element method. The sensitivity of the compliance-Normalized Area relation to perturbation in the location of compliance measurement has also been examined.

\subsection{Finite element model}

Semi-elliptic cracks in cylindrical rod subjected to tension were considered. The finite element package ABAQUS was employed for analysis. Owing to symmetry, only one quarter of the rod was analyzed. Twenty-node isoparametric three-dimensional elements were employed while collapsed quarter point elements [22] were used along the crack tip. The stress/strain fields of a cracked rod with diameter $D=15 \mathrm{~mm}$ and half-rod length $L=45 \mathrm{~mm}$ were computed. The Young's modulus and Poisson ratio of the material were taken to be $200 \mathrm{GPa}$ and 0.3 , respectively. Axial tensile stress was applied to the end of the rod. Two boundary conditions have been considered: an end-free one where the ends are free to rotate and an end-constrained one where the last layer of elements at the rod end was constrained to move in the axial direction without rotation. The element dimensions were gradually refined until the solution converged to a consistent value. For the end-constrained case, the length of the constrained region was also varied to obtain a convergent solution. The constrained end length chosen was $1 \mathrm{~mm}$ for a half-rod length of $45 \mathrm{~mm}$.

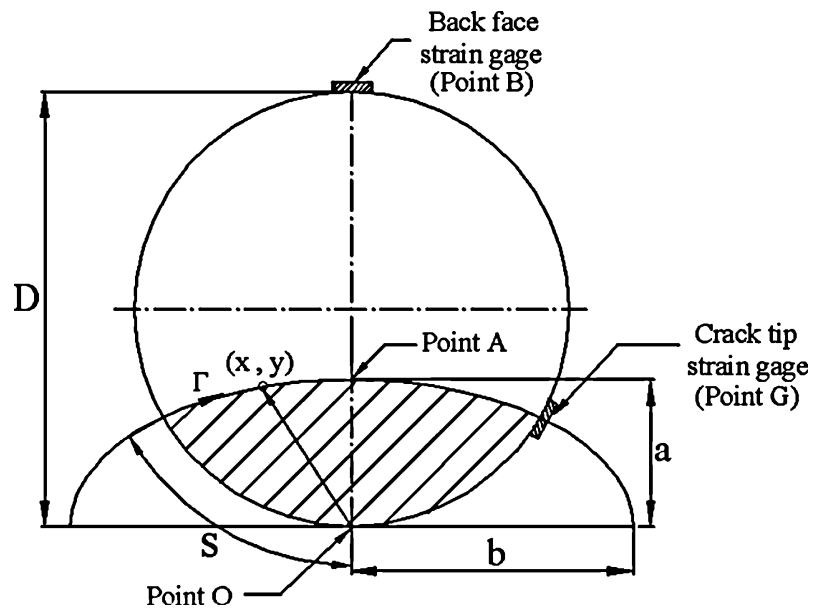

Fig. 1. Nomenclature used for an elliptical surface crack. 
The compliance $C$ of a cracked specimen may be based on any representative displacement which reflects the effect of the crack. In the current work, compliance values for each crack configuration were computed using the back-face strain $\varepsilon_{\mathrm{B}}$ at point $\mathrm{B}$ :

Compliance $\quad C=\frac{\varepsilon_{\mathrm{B}}}{\sigma}$

where $\sigma$ is the nominal axial stress in the rod in MPa.

Besides the back-face strain, the crack mouth opening displacement (CMOD) at point $\mathrm{O}$ (see Fig. 1) can also reflect the specimen compliance. The quantity $\mathrm{CMOD} / \sigma$ will be in direct proportion to the specimen compliance and has been employed in the current work as well.

The Normalized Area (NA) and normalized harmonic mean radius (NHMR) were computed using Eqs. (1) and (2).

\subsection{Effect of crack aspect ratio $a / b$}

Fig. 2(a) shows the compliance obtained from the backface strain versus crack depth ratio $(a / D)$ for different crack aspect ratios $(a / b)$. The crack aspect ratios spanned from a straight edged crack $(a / b=0)$ to a semi-circular crack $(a / b=1)$. As crack depth increases, compliance drops gradually at first. The drop becomes more abrupt when $a / D$ is greater than 0.5 . As the crack aspect ratio $(a / b)$ increases from 0 to 0.3 , there is only a slight change in compliance. Compliance changes more abruptly as crack aspect ratio is approaching unity. Fig. 2(b) and (c) show the above compliance data in terms of Normalized Area and Normalized Mean Harmonic Radius, respectively. It can be seen that in either cases all the data fall nearly onto a single curve. The solid lines in Fig. 2(b) and (c) were obtained by polynomial fitting to all the data. When correlated in terms of the Normalized Area (NA) (Fig. 2(b)), the worst case deviation from the fitted curve is $1 \%$ and it occurs at $a / b=1$. If crack depth under this configuration is to be deduced from the fitted polynomial, an error of $0.11 \mathrm{~mm}$ will occur. When correlated with NHMR (Fig. 2(c)), the maximum deviation from the fitted curve is $1.3 \%$, which corresponds to an error of $0.19 \mathrm{~mm}$ in crack depth. For $a / b \leq 0.6$, the maximum error in derived crack depth is smaller than $0.06 \mathrm{~mm}$. Fortunately, finite element analysis $[18,21]$ and experimental investigations $[2,19,23]$ indicated that the aspect ratio of surface cracks with different initial crack shapes tends to fall between 0.5 and 0.7 when $a / D>0.5$. This corresponds to a maximum error of $0.02 \mathrm{~mm}$. If the compliance based on the crack mouth opening is used (Fig. 3), the above discussion still holds and the degree of accuracy is similar. Thus, it is a matter of convenience how to choose to measure the compliance. In the current experimental work, back-face strain was monitored and compliance will be based only on the back-face strain in the following discussion.
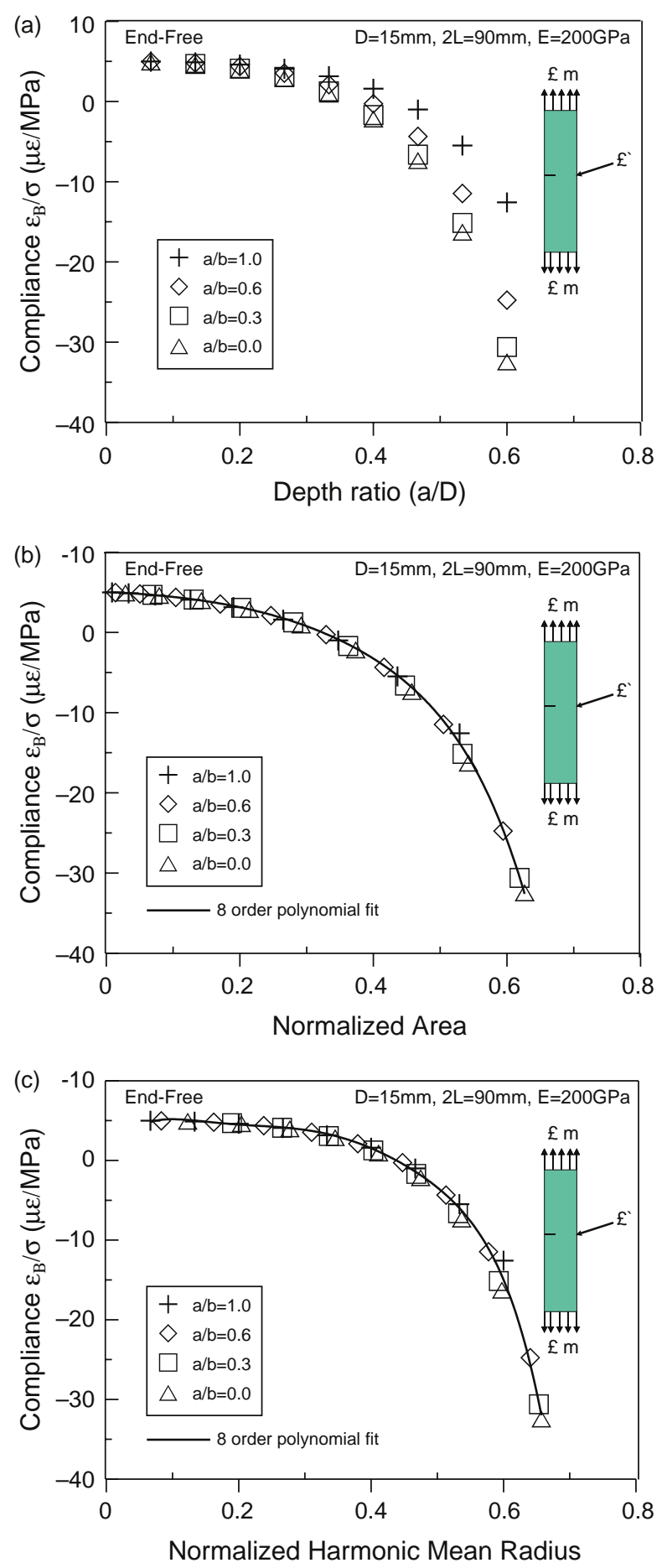

Fig. 2. Back-face compliance for different crack aspect ratios correlated in terms of (a) crack depth ratio; (b) Normalized Area and (c) Normalized Harmonic Mean Radius.

\subsection{Effect of Young's modulus and end constraint}

The compliance of a cracked shaft depends not only on the crack geometry but also on the Young's modulus $(E)$ of the shaft material. However, a single master complianceNA curve can be obtained by introducing a Normalized 

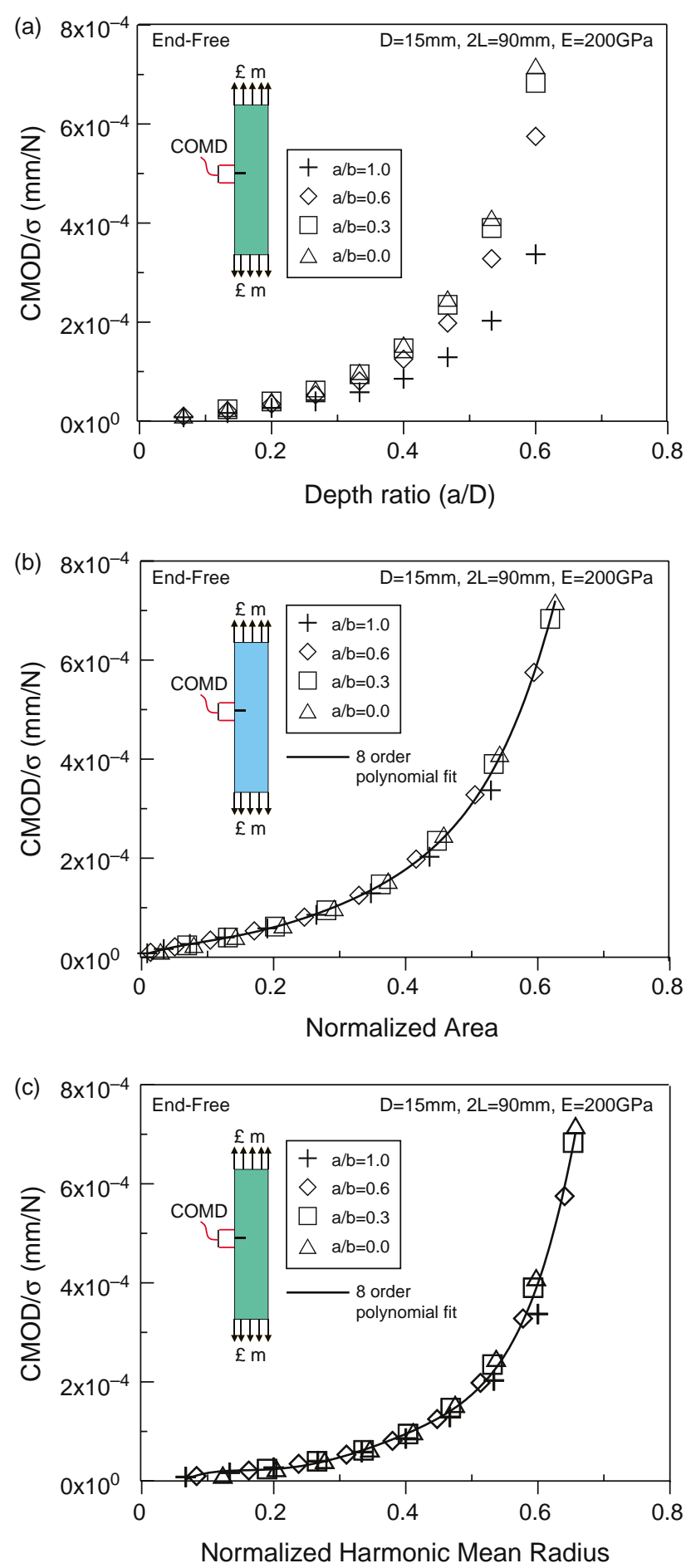

Fig. 3. Crack-mouth compliance for different crack aspect ratios correlated in terms of (a) crack depth ratio; (b) Normalized Area and (c) Normalized Harmonic Mean Radius.

Compliance $X$ :

$X=\frac{E \varepsilon_{\mathrm{B}}}{1000 \sigma}$

where $E$ is in $\mathrm{GPa}$ and $\sigma$ is in MPa.

Fig. 4 shows the finite element results of the Normalized Compliance data for a typical steel specimen $(E=200 \mathrm{GPa})$

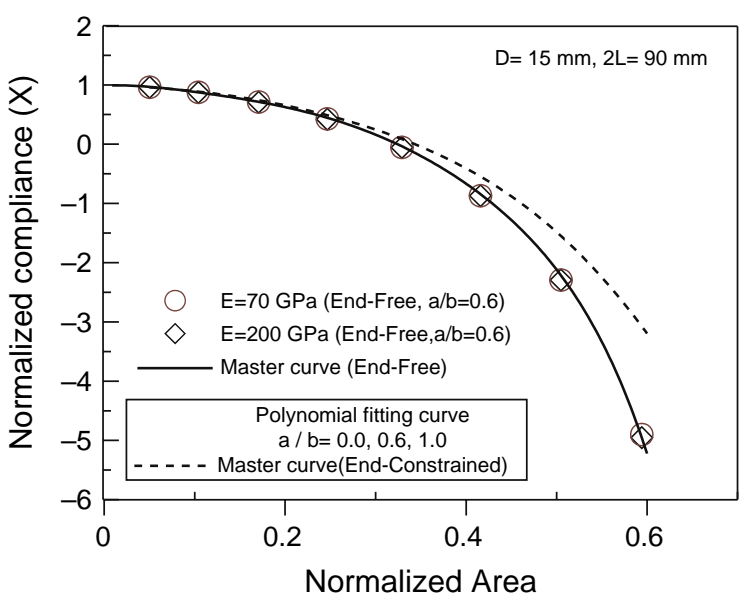

Fig. 4. Effect of Young's Modulus and end constraint on the Normalized Compliance-Normalized Area relationship.

and a typical aluminum alloy specimen $(E=70 \mathrm{GPa})$ for $\mathrm{NA}<0.6$. Both specimens have ends free to rotate. The master curve for this end-free condition can be described by Eq. (5). The degree of curve fitting polynomial was so chosen as to give an error within $0.5 \%$ of the numerical results. With the current description, the maximum deviation of individual data from the master curve is $0.46 \%$.

(i) $-3.0<X \leq 0.95$ or $0.04 \leq \mathrm{NA}<0.54$ (End-free condition):

$$
\begin{aligned}
\mathrm{NA}= & 0.32141-0.13733 X-2.99512 \times 10^{-2} X^{2} \\
& -3.09777 \times 10^{-2} X^{3}-5.35842 \times 10^{-2} X^{4} \\
& -3.6832 \times 10^{-2} X^{5}-1.18526 \times 10^{-2} X^{6} \\
& -1.81867 \times 10^{-3} X^{7}-1.07638 \times 10^{-4} X^{8}
\end{aligned}
$$

(ii) $-17.0<X \leq-3.0$ or $0.54 \leq \mathrm{NA}<0.74$ (End-free condition):

$$
\begin{aligned}
\mathrm{NA}= & 0.40272-5.91933 \times 10^{-2} X-5.30398 \\
& \times 10^{-3} X^{2}-2.62949 \times 10^{-4} X^{3} \\
& -5.15889 \times 10^{-6} X^{4}
\end{aligned}
$$

If the end rotation is constrained in cases such as testing the rod specimen with hydraulic grips, the Normalized Compliance $X$ can be related to the NA by:

(i) $-2.0<X \leq 0.95$ or $0.04 \leq \mathrm{NA}<0.54$ (End-constrained condition):

$$
\begin{aligned}
\mathrm{NA}= & 0.34167-0.174981 X-0.10034 X^{2}+2.62825 \\
& \times 10^{-2} X^{3}+0.15304 X^{4}+1.87761 \times 10^{-2} X^{5} \\
& -0.149421 X^{6}-0.10801 X^{7}-2.21154 \times 10^{-2} X^{8}
\end{aligned}
$$


(ii) $-15.0<X \leq-2.0$ or $0.54 \leq \mathrm{NA}<0.87$ (End-constrained condition):

$$
\begin{aligned}
\mathrm{NA}= & 0.38076-9.48426 \times 10^{-2} X-9.70327 \\
& \times 10^{-3} X^{2}-5.34726 \times 10^{-4} X^{3} \\
& -1.10686 \times 10^{-5} X^{4}
\end{aligned}
$$

The above end constrained curve is shown as broken line in Fig. 4. As expected, the end constrained case has a larger compliance than the end free case at the same NA. This difference become more marked when NA is larger than 0.4.

\subsection{Effect of specimen diameter and length}

Fig. 5(a) compares the compliance-Normalized Area relation for cracked rod specimens with diameters equal to 5,10 and $15 \mathrm{~mm}$. The length $(2 L)$ of the specimen is $90 \mathrm{~mm}$ in all cases. All data fall onto the same curve with negligible discrepancy between different cases.

Fig. 5(b) examines the effect of specimen length. The specimen diameter is kept constant at $15 \mathrm{~mm}$ while the specimen half length to diameter ratio $(L / D)$ varies from 1 to 5. The compliance results for $L / D=3$ and 5 are basically the same. The maximum deviation of the NA at $L / D=1$ from that at $L / D=3$ is $0.9 \%$ and this corresponds to an error of $0.08 \mathrm{~mm}$ in crack depth.

\subsection{Sensitivity of compliance data \\ to the measurement location}

In the current work, the compliance-NA master curve is computed using the back-face strain at the centerline bisecting the crack. In practical monitoring, the strain gage may be stuck off axis due to error in locating the centerline. It is also possible that unevenness may occur in surface crack initiation so that the resulting crack may not be exactly aligned with the expected centerline. Fig. 6 compares some off axis (at an angle $\theta$ ) compliance with that at the centerline for a series of crack depths while the crack aspect ratio was fixed at $a / b=0.6$. The angle $\theta$ is as defined in the inset in Fig. 6. Since the nodal points in the neighborhood of the central point $\mathrm{B}$ are not situated at exactly the same angular position $\theta$ in different meshes, the values of $\theta$ for different crack depths are slightly different. Nevertheless, the comparison in Fig. 6 suggests that strain measurements at an off-axis angle $\theta$ up to $\sim 4^{\circ}$ does not cause any significant error. The maximum error in NA so deduced is $0.3 \%$. It occurs at a value of NA of 0.6 and corresponds to an error of $0.03 \mathrm{~mm}$ in the deduced crack depth. When the off-axis angle increases to $\sim 12-16^{\circ}$ the error in compliance under the same NA increases as expected. The maximum error is associated with the largest off-axis angle at the highest NA, and it corresponds to an error of $0.24 \mathrm{~mm}$ in the deduced crack depth.
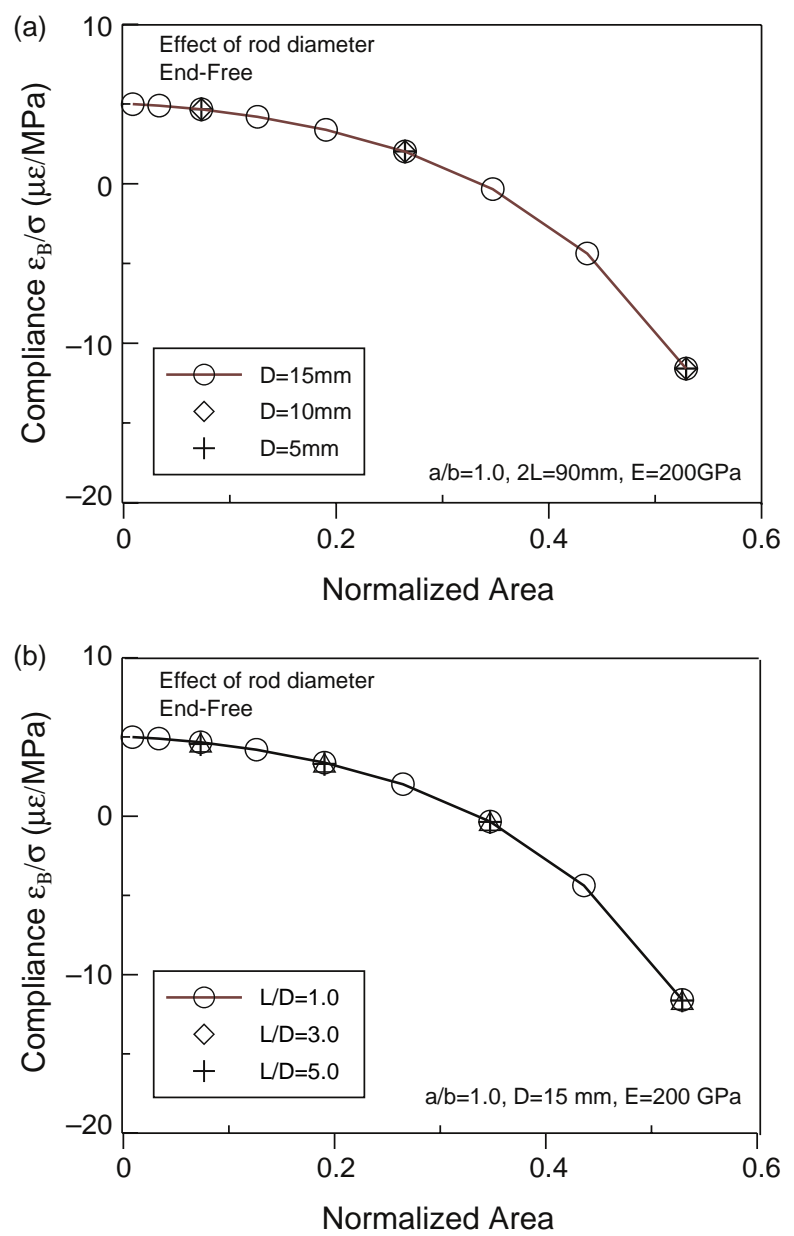

Fig. 5. Effect of (a) rod diameter and (b) specimen length on the Normalized Compliance-Normalized Area relationship.

\subsection{Effect of crack shape}

In the above finite element analysis, a semi-elliptical crack front has been assumed throughout. This crack shape

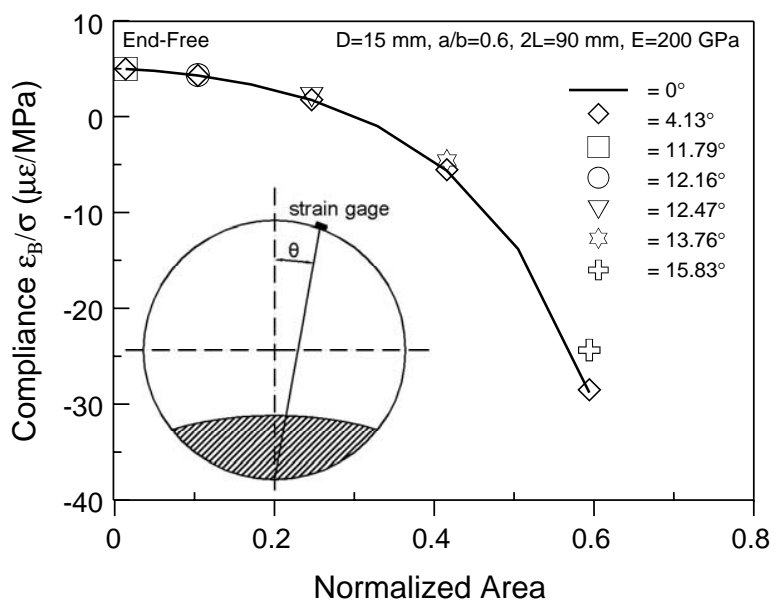

Fig. 6. Effect of off-axis strain gage on the Normalized ComplianceNormalized Area relationship. 


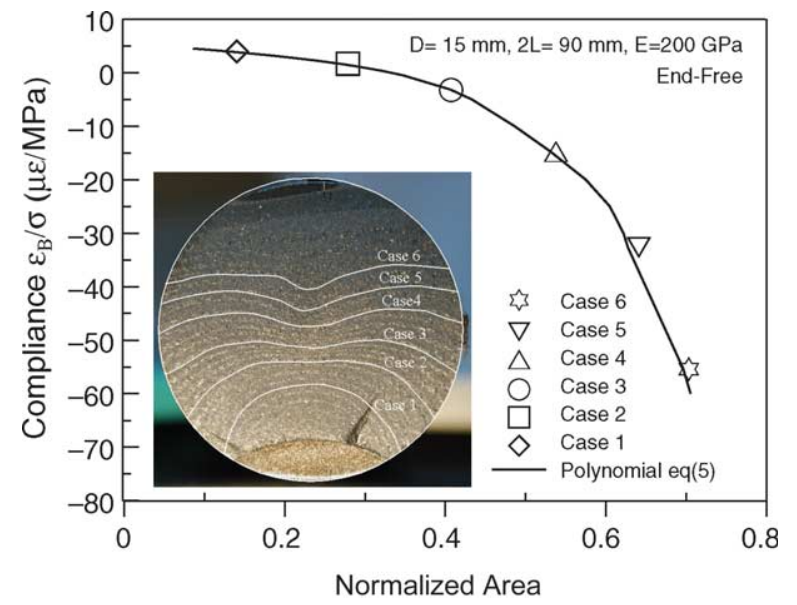

Fig. 7. Effect of deviation from elliptical crack shape on the Normalized Compliance-Normalized Area relationship.

agrees well with a number of independent experimental observations $[2,19]$. However, under certain circumstances, the surface crack front may not 'behave well' and become non-elliptical. For example, the photograph in Fig. 7 shows the fatigue fracture surface of a cold drawn rod that has not undergone stress relief treatment. Residual stress in the rod makes the crack front deviate from semi-elliptical shape as the crack depth increases. The crack fronts, designated case $1-6$, have been digitized and input to the finite element model to calculate their respective compliance. The compliance versus NA data is compared against those for an elliptical crack. Maximum discrepancy in NA occurs in case 5, which deviates from the elliptical case by $1.3 \%$. From this example it can be seen the relationship between compliance and NA is quite robust. The difference is not marked even where there is large deviation in crack shape from being elliptical. However, under this situation, the deduction of crack depth is rather meaningless. This is a limitation common to all indirect methods for deducing the surface crack dimension.

\section{Comparison with experimental measurements}

\subsection{Experimental procedure}

Cylindrical rod specimens with length $2 L=200 \mathrm{~mm}$ and diameter $D=14.97 \mathrm{~mm}$ were machined from $15 \mathrm{~mm}$ diameter AISI 304 stainless steel rod stock (Exp 1-5) and $18 \mathrm{~mm}$ thickness AISI 304 stainless steel plate stock (Exp 6-8), respectively. Semi-elliptical surface starter notches with different aspect ratios were created on the rod specimen via electric discharge machining using a thin circular copper foil electrode. To relieve the residual stresses before testing, Specimen Exp 4-8 have been solution treated in vacuum at $1050{ }^{\circ} \mathrm{C}$ for one hour followed by quick cooling in a continuous supply of argon stream.

The rod specimens were tested with a pair of rigid hydraulic grips to simulate the end-constrained condition.
In all cases, a gripping length of $35 \mathrm{~mm}$ was adopted at each end. Surface crack length was monitored with a rotary traveling microscope to a resolution of $0.026 \mathrm{~mm}$ on a $15 \mathrm{~mm}$ diameter specimen. On all specimens, instantaneous crack fronts were recorded periodically by creating beach marking using a waveform that has the same maximum but with the amplitude reduced to $30 \%$ of the instantaneous baseline loading. Load shedding was applied to Exp 1, Exp 2 and $\operatorname{Exp} 4$ to maintain a constant $\Delta K$ condition at the deepest interior point $\mathrm{A}$. Constant amplitude loading was employed for the other specimens. A loading ratio of 0.1 was used for all loading cases.

\subsection{Effect of crack closure}

In a real life fatigue crack, premature crack closure will occur. For a surface crack, the phenomenon of crack closure is highly complex [25-28]. Crack closure is heavier on the surface than in the interior. The overall compliance of the specimen will be affected as crack closure develops. In fact, compliance change aided by an offset procedure is a widely used technique to measure the point of crack opening and closure [24-26]. This technique is employed in the current work. A strain gage straddling the crack at the tip region (Point G) was used to reflect the crack closure on the surface. A back-face gage, sticking at point B in Fig. 1, was used to reflect the bulk closure. This bulk closure is more representative of the closure in the interior $[25,26]$. On unloading, the slope of the back-face compliance trace starts to change as closure at the surface begins to occur. It is continuously changing as closure spreads from the surface to the interior. The point on the compliance trace that corresponds to a fully closed crack is taken as the crack closure load for the deepest interior point [27].

A typical compliance trace of normalized load versus normalized back-face strain is shown in Fig. 8. Two marked turning points are evident. If the offset procedure [24,25] is used, the turning points are even more well-defined.

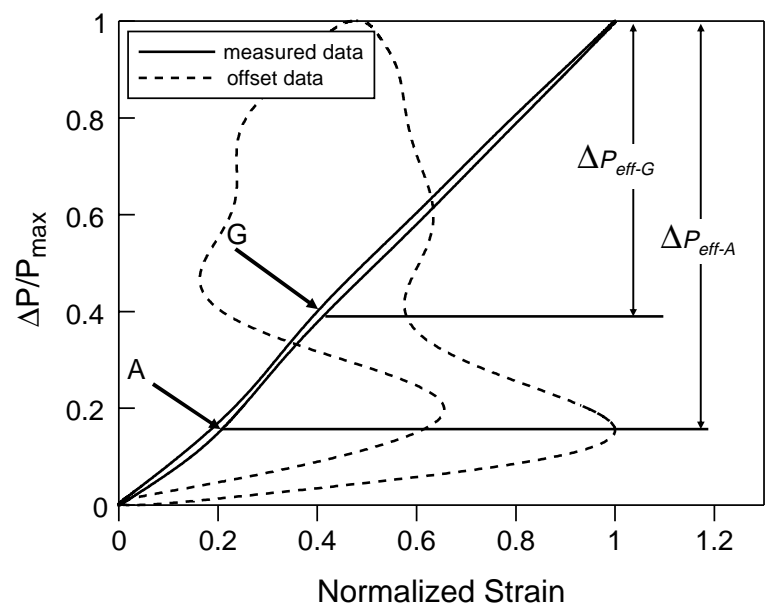

Fig. 8. Typical compliance trace for identification of crack closure. 
These turning points may be attributed to the closure of crack at the surface point $\mathrm{G}$ and the deepest interior point $\mathrm{A}$, respectively. The plane stress condition prevailed near the specimen surface will lead to a heavier plastic deformation and an earlier closure at the surface point $G$ than that at the interior point A. Dawicke et al. [27] and Troha et al. [28]

(a)

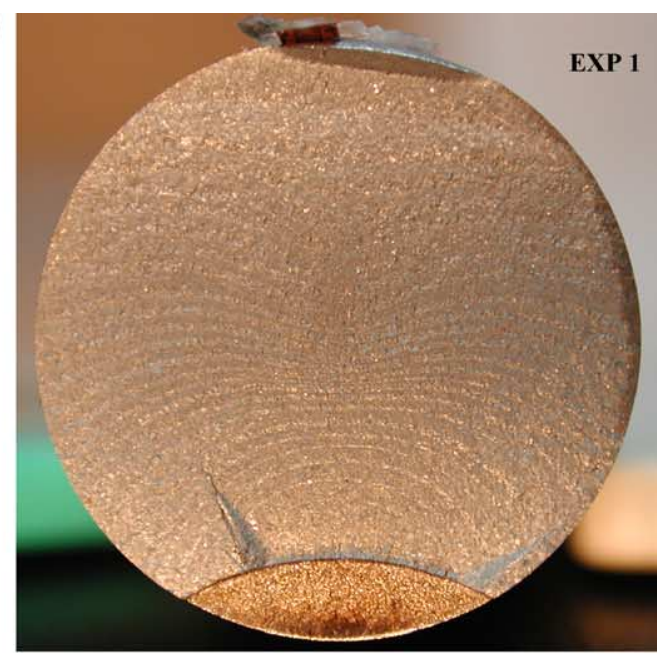

(b)

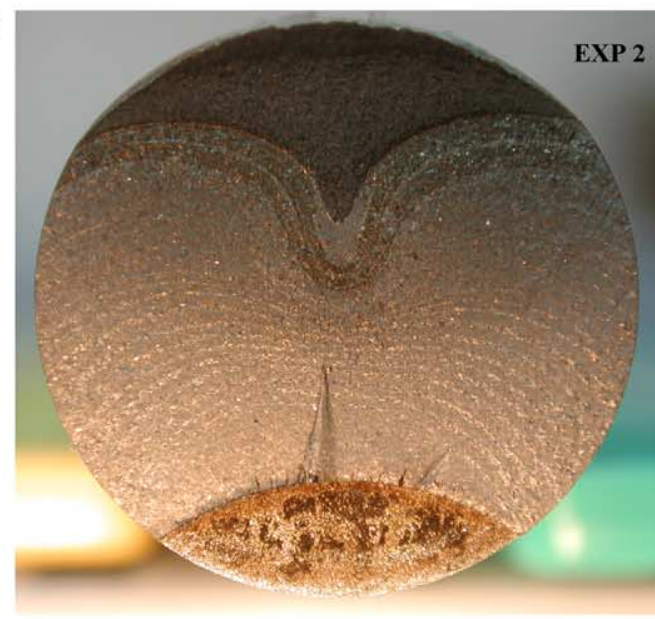

(c)

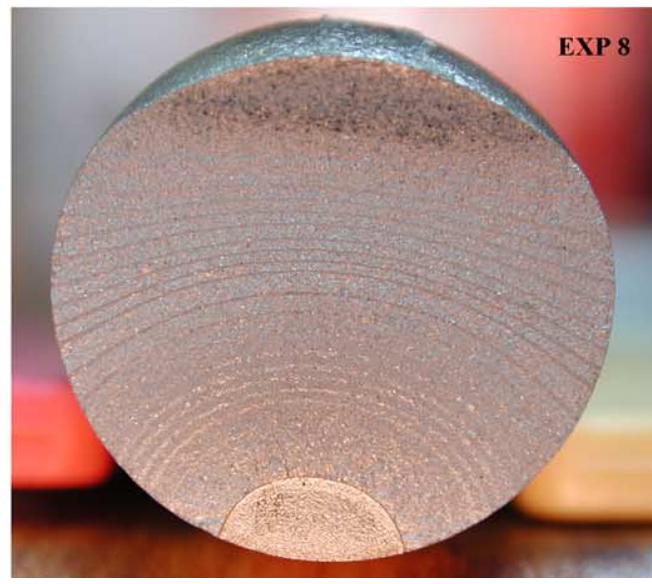

Fig. 9. Fracture surface appearance of (a and b) as received; (c) solution annealed specimens to show the effect of stress-relief heat treatment on the development of crack shapes. also observed a similar phenomenon. In the presence of crack closure, it is clear that the compliance used for crack length deduction must be carefully chosen from the compliance trace. In the current work, compliance data were computed from the unloading portion of the load-strain curve above the first turning point.

\subsection{Effect of residual stress}

It has been pointed out before that residual stress in the specimen will have a great impact on the development of crack shape. In the current experimental work, the first specimens were tested in the as-received condition and highly irregular crack shapes resulted (see Exp 1 and Exp 2 in Fig. 9). Later on solution anneal treatment was applied to relief the residual stresses before testing and much more regular crack shapes were obtained (see Exp 8 in Fig. 9).

\subsection{Comparison between numerical and experimental results}

In the current experimental set-up, the hydraulic grips constrained the end rotation of the specimens. Experimentally measured compliance versus NA curve and finite element results are both presented in Fig. 10. As expected, at small crack size, the end-free and end-constrained results do not differ significantly. These two results become distinct for $\mathrm{NA} \geq 0.4$. On the other hand, experimental data in general agree well with the numerical results for the endconstrained case for NA smaller than 0.7. Above an NA of 0.7 , experimental data from specimens tested under constant amplitude loading (Exp 3 and Exp 5-8) start to deviate from the FEM results. Data from specimens tested under the load shedding condition (Exp 1, Exp 2 and Exp 4) show distinct deviation from the FEM results only at NA $>0.85$. Such deviations are probably caused by the extensive plastic zone in the remaining ligament. The degree of plastic deformation is reduced under a decreasing load test and so

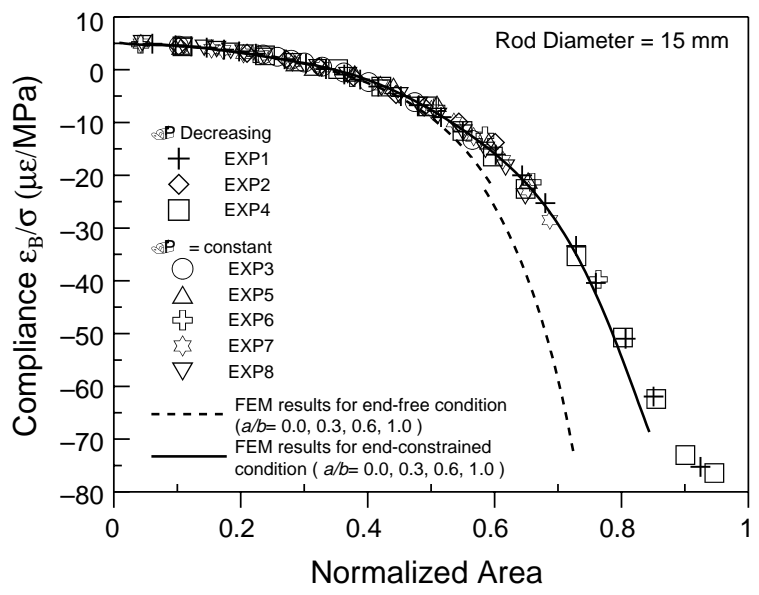

Fig. 10. Comparison of experimental and FEM compliance-Normalized Area results. 
(a)
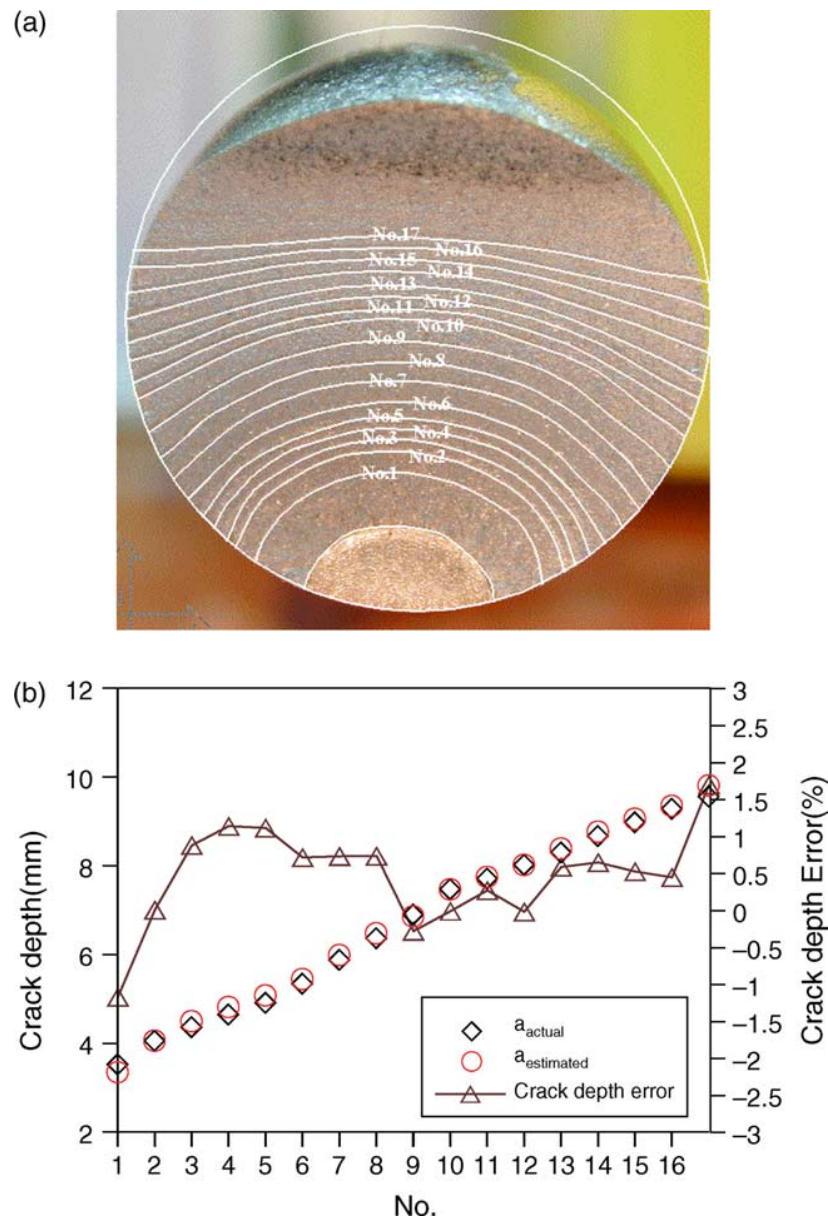

Fig. 11. (a) highlighted beach marking and (b) comparison between predicted and measured crack depths in specimen Exp 8.

the corresponding deviation from the elastic FE analysis is prominent only at a larger NA. Within the typical data acquisition range $(0.1<\mathrm{NA}<0.7)$ in a fatigue crack propagation test, the differences between experimental and numerical NA are typically smaller than 0.012 under the same compliance. In the worst case, the maximum difference in NA is 0.076 and it occurs at NA $=0.85$. It is interesting to note that the measured compliance-NA relationship holds well even for non- elliptical crack shapes (Exp 1 and 2, see Fig. 9).

Fig. 11(a) shows the highlighted beach marking on the fracture surface of a typical specimen (Exp 8). Fig. 11(b) compares the measured crack depth for each of the corresponding beach markings with the crack depth predicted using the compliance and surface crack length. Errors tend to be larger at the beginning and at the end of the test. In the former case, the crack shape has not yet been well developed. In the latter, increasing degree of plastic deformation and deviation from the assumed elliptical shape contribute to the increasing error. Nevertheless, the majority percentage errors are within $\pm 1 \%$ and the worst-case error is $1.7 \%$. The average percentage error among all the stress

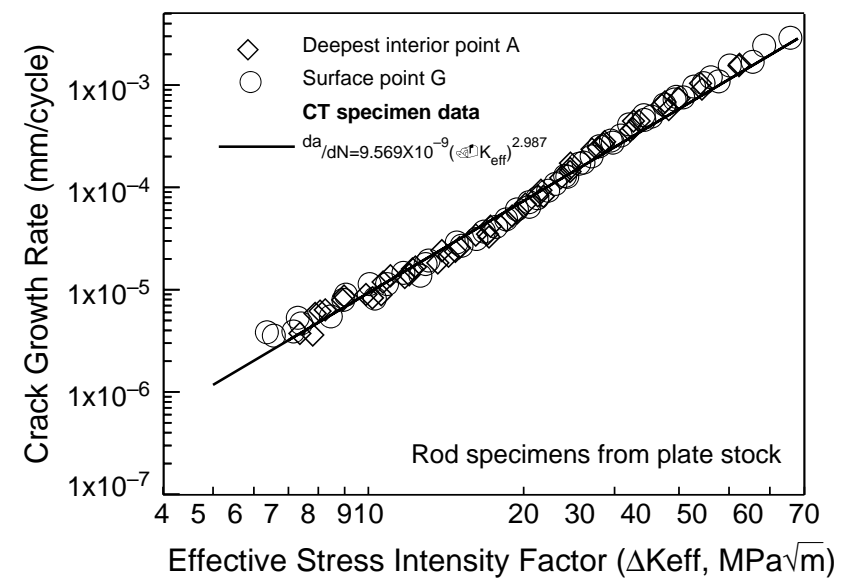

Fig. 12. Comparison of fatigue growth rates of surface cracks in four cylindrical rods with those of long cracks in three standard compact tension specimens.

relieved specimens tested is $0.57 \%$, which corresponds to an average error of $0.082 \mathrm{~mm}$ in crack depth.

Finally, Fig. 12 compares the fatigue crack growth rates of surface cracks in four cylindrical rods with those of the long through cracks in three compact tension specimens in terms of the effective stress intensity ranges. Crack growth rate at the surface point $G$ is derived from surface crack length measurement. Growth rate at the deepest interior point $\mathrm{A}$ is derived from the crack depth inferred from the compliance method mentioned above. The effective stress intensity ranges are calculated using the closed form stress intensity solution reported elsewhere [2] and taking into account of crack closure. It can be seen that the surface crack growth results from both the surface and the deepest points agree with each other and with the compact tension data as well. Thus the direct prediction of crack depth and the indirect comparison through the crack growth data both suggest that the compliance method for deducing the surface crack depth is a viable technique.

\section{Conclusions}

(1) The compliance versus the Normalized Area relationships for a cracked cylindrical rod under tension are practically independent of the crack aspect ratio, crack depth, Young's modulus, specimen diameter, crack shape and specimen length provided the specimen half length to diameter ratio is larger than or equal to 3 .

(2) The compliance versus Normalized Area relationship is significantly affected by the end constraint condition of the specimen. It is also affected by specimen length if the specimen half length to diameter ratio is smaller than unity.

(3) The experimental compliance versus Normalized Area relationship agrees with the curve derived using finite element analysis. 
(4) If the crack profile is semi-elliptical, by measuring the compliance and the surface crack length, the crack depth can be deduced from a compliance-NA master curve to within $2 \%$ or $0.082 \mathrm{~mm}$ of the measured depth on average.

(5) Crack growth data deduced from this crack depth measurement agrees well with the crack growth data obtained from the standard compact tension specimen.

\section{Acknowledgements}

The authors are grateful to the National Science Council, R.O.C. for financial support for this work through the projects NSC 92-2212-E-002-008 and NSC93-2212-E-002-062.

\section{References}

[1] Shin CS, Chen PC. Fatigue crack propagation testing using subsized rotating bending specimens. Nucl Eng Des 2004;(231):13-26.

[2] Shin CS, Cai CQ. Experimental and finite element analyses on stress intensity factors of elliptical surface crack in a circular shaft under tension and bending. Int J Fract 2004;(129):239-64.

[3] Wilhem D, FitzGerald J, Carter J, Dittmer D. An empirical approach to determining $\mathrm{K}$ for surface cracks. Proceedings of the fifth international conference on fracture research, 1982. p. 11-21.

[4] Ragazzo C, Hertzberg RW, Jaccard R. A method for generating fatigue marker bands using a constant $K_{\max }$ test procedure. J Test Eval 1995;(23):19-26.

[5] Varvani-Farahani A, Topper TH. Short fatigue crack characterization and detection using confocal scanning laser microscopy (CSLM). Nontraditional method of sensing stress, strain, and damage in materials and structures. ASTM STP 1997;1318:43-55.

[6] Saka M, Sato I, Abe H. NDE of 3-D surface crack using magnetic field induced by DC current flow. NDT E Int 1997;31:325-8.

[7] McFadyen NB, Bell R, Vosikovsky O. Fatigue crack growth of semielliptical surface cracks. Int J Fatigue 1990;(12):43-50.

[8] Okada H, Zhao W, Atluri SN, Sampth SG. A computational approach to determining the depth of surface flows by the ACPD technique. Eng Fract Mech 1992;43:911-21.

[9] Bian LC, Lim JK. Fatigue life prediction of the plates with an inclined surface crack. Int J Fatigue 2002;(25):521-31.
[10] Ikeda K, Yoshimi M, Miki C. Electrical potential drop method for evaluating crack depth. Int J Fract 1991;(47):25-38.

[11] Harrington DS, Bell R. A mobile DCPD probe arrangement for improved crack profile measurements. Br J NDT 1993;(35):497-501.

[12] Harrington DS, Bell R, Tan CL. Calibration of the localized DCPD method for crack shape measurement using the boundary element method. Fatigue Fract Eng Mater Struct 1995;(18):875-84.

[13] Saka M, Oouchi A, Abe H. NDE of a crack by using closely coupled probes for DCPD technique. ASME J Pressure Vessel Technol 1998; (118):198-202.

[14] Yee R, Lambert SB. A reversing direct current potential drop system for detecting and sizing fatigue cracks along weld toes. J Test Eval 1995;(23):254-60.

[15] Romilly DP. Development of the DC potential drop method for oneand two-directional crack growth monitoring. Exp Tech 1990;(14): $22-8$.

[16] Beevers CJ. Advances in crack length measurement. London, UK: EMAS; 1982.

[17] Shin CS, Huang WH, Chen HY. An improved de potential drop system for crack length measurement. J Chin Inst Eng 1993;(16): $29-40$.

[18] Carpinteri A, Brighenti R. Part-through cracks in round bars under cyclic combined axial and bending loading. Int J Fatigue 1996;(18): 33-9.

[19] Caspers M, Mattheck C, Munz D. Propagation of surface cracks in notched and unnotched rods. Surface-crack growth: models, experiments, and structures. ASTM STP 1990;1060:365-89.

[20] Ikeda K, Yoshimi M, Miki C. Electrical potential drop method for evaluating crack depth. Int J Fract 1990;(47):25-38.

[21] Lin XB, Smith RA. Shape growth simulation of surface cracks in tension fatigued round bars. Int J Fatigue 1997;(19):461-9.

[22] Barsoum RS. On the use of isoparametric finite element $s$ in linear fracture mechanics. Int J Numer Methods Eng 1976;(10):25-37.

[23] Lorentzen T, Kjaer NE, Henriksen T. Application of fracture mechanics to surface cracks in shafts. Eng Fracture Mech 1985; (23):1005-14

[24] Kikukawa M, Jono M, Tanaka K. Fatigue crack closure behaviour at low stress intensity level. Proc ICM2, Boston, 1976. p. 254-277.

[25] Fleck NA. Influence of stress state on crack growth retardation. Basic Questions in Fatigue, ASTM STP 924, 1988. p. 157-83.

[26] Fleck NA, Smith IFC, Smith RA. Closure behaviour of surface cracks. Fatigue Fract Eng Mater Struct 1983;(3):225-39.

[27] Dawicke DS, Grandt Jr AF, Newman Jr JC. Three-dimensional crack closure behavior. Eng Fract Mech 1990;36:111-21.

[28] Troha WA, Nicholas T, Grandt Jr AF. Three dimensional aspects of fatigue crack closure in surface flaws in polymethylmethacrylate material. ASTM STP 1988;982:598-616. 